\title{
Video Article \\ Monitoring Colony-level Effects of Sublethal Pesticide Exposure on Honey Bees
}

\author{
William G. Meikle ${ }^{1}$, Milagra Weiss ${ }^{1}$ \\ ${ }^{1}$ Carl Hayden Bee Research Center \\ Correspondence to: William G. Meikle at William.Meikle@ars.usda.gov \\ URL: https://www.jove.com/video/56355 \\ DOI: doi: $10.3791 / 56355$
}

Keywords: Environmental Sciences, Issue 129, Hive evaluation, hive weight, hive temperature, honey bee colony phenology, imidacloprid, neonicotinoid

Date Published: 11/15/2017

Citation: Meikle, W.G., Weiss, M. Monitoring Colony-level Effects of Sublethal Pesticide Exposure on Honey Bees. J. Vis. Exp. (129), e56355, doi:10.3791/56355 (2017).

\section{Abstract}

The effects of sublethal pesticide exposure to honey bee colonies may be significant but difficult to detect in the field using standard visual assessment methods. Here we describe methods to measure the quantities of adult bees, brood, and food resources by weighing hives and hive parts, by photographing frames, and by installing hives on scales and with internal sensors. Data from these periodic evaluations are then combined with running average and daily detrended data on hive weight and internal hive temperature. The resulting datasets have been used to detect colony-level effects of imidacloprid applied in a sugar syrup as low as 5 parts per billion. The methods are objective, require little training, and provide permanent records in the form of sensor output and photographs.

\section{Video Link}

The video component of this article can be found at https://www.jove.com/video/56355/

\section{Introduction}

Concerns have been raised recently about the exposure of honey bees to low concentrations of agrochemicals, particularly pesticides. Honey bees in the field may be exposed via direct application on foragers in the field or even the colonies themselves, by contact with treated surfaces, by dust from seed treatments, and by consumption of plant products such as pollen, nectar, and exudate ${ }^{1,2,3,4,5}$. Acute toxicity is clearly detrimental to honey bees, but the concentrations that causes $50 \%$ mortality in test insects ( $\mathrm{LC}_{50}$ ) of many pesticides, including neonicotinoids such as imidacloprid, are far higher than that observed in bee bread and wax ${ }^{6}$ and higher than what is considered a "field realistic" range ${ }^{7}$. However, recent work has shown significant effects of sublethal exposure to pesticides on the colony level ${ }^{8}$, even for concentrations as low as 5 parts per billion $(\mathrm{ppb})^{9}$.

Honey bee colonies have been referred to as "superorganisms" because many functions associated with an individual animal, including food procurement, reproduction (which honey bee colonies conduct via colony fission), and temperature control, are conducted by groups of adult worker bees ${ }^{10,11,12,13,14}$. Honey bee colonies, considered as organisms, offer a unique opportunity for study because the structures that support the colonies can be disassembled, examined, and reassembled with, if done carefully, little, or no adverse effects. Beekeepers and researchers take advantage of this by disassembling hives to inspect them for, among other things, the presence and status of the queen, pests, and pathogens, sufficiency of food stores, quantity of brood, and other factors ${ }^{15}$. Bee colonies have another important characteristic in that, unlike most homeotherms, they are typically stationary and obtain resources by deploying forager bees that can collect food at distances up to $10 \mathrm{~km}^{16}$. Bee hives can thus be maintained indefinitely on electronic scales, and the resulting continuous weight data has been shown to provide information on honey storage, foraging activity, foraging success, swarming, syrup consumption, lethal and sublethal pesticide effects, pest infestation and robbing $9,17,18,19,20$. Because all parts of the colony, including brood, can be accessed, sensors for temperature, humidity, $\mathrm{CO}_{2}$, sound, and other factors can be installed within the hive and used to obtain continuous data which inform on the health and status of the colony ${ }^{21}$.

Estimates of the size of the adult bee and brood populations are useful if not critical for interpreting many kinds of continuous data. Visual assessments are fast and widely applied in field studies ${ }^{22}$ but have shortcomings. Visual inspections are usually conducted during the day and thus do not account for foraging bees, which can compose a significant part of the population ${ }^{23}$. The precision and accuracy of estimates from visual inspections also depend on the training and experience of the inspector(s). Objectivity is another consideration, particularly if the hive inspectors are aware of which hives are in which treatment groups; assumptions about the distribution of the error of such measures may be difficult to justify.

Rate of hive weight gain, size of the foraging population, and within-hive temperatures, to provide a few examples, would be expected to be quantitatively different between large and small colonies, so measuring and controlling for colony size often greatly facilitates the detection of treatment effects in randomized experiments. For research purposes, a precise and objective measure of the adult bees was developed based in the principle that the difference between the total hive weight during a period of inactivity, such as at night, and the sum of the weight of all the 
hive parts (including woodenware, wax, honey, and brood) is the weight of the total adult bee mass. By weighing frames and measuring brood mass using frame photographs, food resources can likewise be estimated by subtracting the brood mass from the frame weight, after controlling for the weights of the empty frame and the wax. These methods are intended to improve on the precision expected from visual assessments in the field.

Protocol

The protocols described below follow the animal care guidelines of USDA Agricultural Research Service.

\section{Full Evaluation}

NOTE: A full evaluation is necessary to determine 1) the weights of all hive parts, including the lid, cover, boxes, bottom board, frames, and 2) the amounts of brood and food resources. The weights of all hive parts will be summed and subtracted from the total hive weight when all bees were present to yield the weight of all the adult bees. Data on the weights of hive parts that are largely constant, such as the lid, bottom board and box(es), can be retained and used in future evaluations, thus expediting the evaluations. Conduct a full evaluation at or near the beginning of the study. Wooden parts may change in terms of moisture content ${ }^{24}$ so repeat full evaluations are recommended after long periods. Weigh a part of the hive, such as the lid, at each evaluation to estimate changes in other hive parts. Place a screened vestibule around the workers and hive during evaluation if other hives are close by and robbing pressure is a concern.

1. Weigh all hive parts. Use a scale with a precision of $1 \mathrm{~g}$ or less.

1. Weigh the lid, inner cover, queen excluder, syrup feeder, and any other parts on the top of the hive.

2. Using a spatula or hive tool, remove, and weigh any food or treatment material, such as protein supplement or grease patty, resting on the tops of the frames. Replace afterward if necessary.

3. Place an empty hive box (hereafter the "temporary box") next to the hive to serve as the transitional location for the frames. Place the temporary box on top of a flat surface, such as a lid or a bottom board, to prevent bees, and particularly the queen, from falling out of the box onto the ground.

4. Move the frames rapidly and gently, preferably in groups of two or three to retain parts of the bee cluster, to the temporary box so the frames are in the same order as they were in the original box. Once all the frames have been moved, shake any remaining bees onto the frames in the temporary box and weigh the hive box.

5. If the box was the lowest box, weigh the bottom board, entrance reducer, and any other hive parts after shaking the bees into the temporary box. Number frames with an indelible marker before moving them so they can be replaced in their original orientation.

6. Reinstall the bottom board, entrance reducer, brood box, and other equipment. Move the frames from the temporary box back to the original brood box in their original order and orientation, again taking care to avoid unnecessary disturbance of the bees.

2. Remove a frame from one end of the box and visually inspect both sides of the frame for the presence of the queen. If the queen is found, place her gently in a part of the box that has already been evaluated, to avoid further disturbance. Shake worker bees gently but firmly into the box between frames, rather than on top, if possible.

3. Weigh the frame and photograph both sides of the entire frame with a digital camera, getting as close as possible to the frame so capped brood and honey cells are easily distinguished, but at a sufficient distance so the entire frame is included. Photograph the frame while another worker holds it.

1. Use a 'frame holder' (any jig that holds the frame upright while on the scale and does not mar the comb) on the scale. Weigh the withinhive syrup feeders.

Note: The size of the frame in the photograph will be used to convert surface areas from relative to absolute measures.

4. Replace the frame in its original position and orientation in the hive box. Remove the next frame prior to reinstalling the previous frame to provide more space to safely shake bees within the box. Check for the presence of the queen, shake to remove bees, weigh, photograph, and replace each subsequent frame until all frames have been processed.

5. If the hive has two or more boxes, weigh and photograph frames from the lowest box first.

1. Remove the upper box or boxes sequentially, stacking them next to the hive in reverse to their original order. Once the bottom box has been evaluated, place the next box to be evaluated on top of the bottom box, place the temporary box on top of the remaining unevaluated boxes, and move frames from the second box to the temporary box, as above. Move the frames back to their original box, in their original orientation.

NOTE: By inspecting the lower boxes first, shaken bees will fall into a hive box that will no longer be disturbed.

6. Obtain the total hive weight during a recent period of inactivity, such as the night or the early morning before the bees are flying on the day of the evaluation. This weight represents the entire colony including the foragers.

7. Calculate the adult bee mass by summing the weights of all the hive parts (woodenware, frames, bee feed, etc.) and then subtracting that sum from the "total hive weight" value obtained in the previous step. The difference between the two is an estimate of the adult bee mass. NOTE: Once the full evaluation is conducted, subsequent evaluations can be conducted on a partial basis, assuming the non-frame hive parts, such as the boxes and the bottom board, have not changed appreciably in weight. Partial evaluations take less time.

8. To conduct a partial evaluation for a hive with a single box, remove, photograph, weigh, and replace the frames without moving frames to a temporary box. In a hive with multiple boxes, the follow the procedure as described above (step 1.5), but without weighing the hive boxes.

\section{Installation of Hives on Scales}

NOTE: The hive scales used in this study are outdoor electronic bench scales with a maximum capacity of at least $100 \mathrm{~kg}$, a load cell precision of about one part in 5000 with temperature compensation, and a pan size of $460 \mathrm{~mm} \times 610 \mathrm{~mm}$. These scales are connected to a 24 VDC 
indicator and a 12-bit datalogger. Indicator output may be digital or analog; scale systems used here had output in mA and required calibration curves to convert data to $\mathrm{kg}$.

1. Install scales on a firm, level surface.

NOTE: Elevating the scales from the ground using concrete or ceramic blocks, or pieces of kiln-dried wood, will keep the scale and electrical connections from coming into contact with soil or water.

2. Cover the scale pan on top with plastic, cloth, cardboard, or other material to prevent excessive light reflection and heating of the hive entrance.

3. Ensure that entrances to hives that are close together face different directions to reduce drift among colonies.

4. Calibrate hive scales upon installation and periodically thereafter, particularly if the scales are moved.

1. Place scale calibration weights on the weighing pan and record the scale output. Ensure that the total weights exceed $50 \%$ of the maximum capacity of the scale.

NOTE: If the scale output is voltage or current units, rather than mass units, then use the slope and intercept of the calibration curve (relationship between total known weight on the pan and scale output) to convert scale output to mass units.

\section{Installation of Temperature Sensors}

NOTE: Temperature sensors used in this study were of two types: 1) thermocouples attached to cables directly connected to a battery-powered datalogger (which may be placed inside or outside the hive, if the cable is long enough); and 2) small, battery-powered devices with integrated sensors and dataloggers and which required removal from the hive for data to be downloaded.

1. Obtain a temperature sensor that can fit between frames, is resistant to the high humidity conditions in the interior of a hive, and has sufficient battery power and memory (or is accessible, via cable or wireless connection).

2. Place sensors that might be affected by wax or propolis in a protective container, such as a tissue embedding cassette (small, disposable plastic box with a snap-shut lid and with ventilation slots). The protective container reduces wax and propolis build-up that can clog sensor access ports of sensors or otherwise affect data download.

3. Attach the sensor just beneath the top bar in the center of the box to provide informative data on brood rearing (see below). NOTE: A short piece of wire is attached to the container with the sensor and the wire stapled to the top of the frame, so the container hangs down one side. Sensors placed directly on the brood mass may interfere with brood and comb maintenance, and sensors placed on frames near the exterior, or placed on inner covers or lids, may be affected a great deal by external conditions, particularly during cool weather.

\section{Preparation of Syrup Treatment}

NOTE: Make treatment solutions fresh the day of each feeding using analytical-grade imidacloprid. Imidacloprid is highly water soluble and therefore easily incorporated in syrup; some pesticides have low water solubility and should be applied using other means. Formulations of $1 \mathrm{~kg}$ of treatment solution were applied using a $1 \mathrm{~L}$ plastic bottle as follows:

1. Mix control (no pesticide) sucrose solution 1:1 w:w (e.g. $500 \mathrm{~g}$ sucrose:500 mL distilled water). Dissolve sucrose in distilled water using a large mixing flask with mixing bar on a hot plate heated to no more than $60^{\circ} \mathrm{C}$.

2. For solutions that will include imidacloprid, mix sucrose solution as above, but withhold $100 \mathrm{~mL}$ (thus "short") to allow for the added volume of respective imidacloprid "spike" solutions.

1. Dissolve $500 \mathrm{~g}$ of sugar in $400 \mathrm{~mL}$ of distilled water to allow for the addition of a $100 \mathrm{~mL}$ spike to achieve $1 \mathrm{~kg}$ of treatment solution. For convenience in the field, transfer $900 \mathrm{~g}$ of "short" sugar solution to another bottle, then add the spike to each individual bottle.

3. Prepare a $10 \mathrm{ppm}$ imidacloprid stock solution by measuring $1.0 \mathrm{mg}$ of pesticide on an analytical balance and dissolving it in $100 \mathrm{~mL}$ of distilled water using a mixing bar without heat.

NOTE: To avoid problems with static electricity, weigh the imidacloprid into small, nonreactive plastic receptacles and place those receptacles directly in the solution. Stir the solution and remove the receptacles after the imidacloprid has dissolved.

4. For a $5 \mathrm{ppb}$ solution, $\operatorname{mix} 0.5 \mathrm{~mL}$ of the stock solution into $99.5 \mathrm{~mL}$ of distilled water to achieve $100 \mathrm{~mL}$ of spike solution. Add this to $900 \mathrm{~g}$ of the short sucrose solution to achieve $1 \mathrm{~kg}$ of $5 \mathrm{ppb}$ syrup. For the $20 \mathrm{ppb}$ solution, mix $2.0 \mathrm{~mL}$ of stock solution into $98.0 \mathrm{~mL}$ of distilled water to produce the $100 \mathrm{~mL}$ spike solution. For a $100 \mathrm{ppb}$ solution mix, $10.0 \mathrm{~mL}$ of stock solution into $90.0 \mathrm{~mL}$ of distilled water for the spike solution.

\section{Preparation of Bee Hives and Application of Treatment}

1. Evaluate colonies to determine the adult bee and brood populations prior to and after application of treatment. Sample material(s) of interest, such as honey, bee bread, bees, or wax, to determine baseline pesticide levels prior to treatment.

1. To sample wax, open a $50 \mathrm{~mL}$ centrifuge tube, select a section of empty comb, and scrape the open mouth of the tube along the comb until the desired amount of wax has been collected. Avoid touching the wax in the tube. Replace the cap on the tube and label the tube. Replace the frame in the hive.

2. To sample honey or nectar, open a centrifuge tube and press the mouth of the tube against the section of comb containing the honey or nectar. Allow the material to flow into the tube, rather than scraping the tube, to reduce the amount of wax in the sample. Replace the cap on the tube and label the tube. Replace the frame in the hive.

3. To sample bee bread, select a frame containing bee bread and use a clean metal or plastic spatula to remove the contents of several cells of bee bread and place the material in a centrifuge tube. Replace the frame in the hive. 
NOTE: Collecting sufficient material will usually take several minutes per frame. A typical sample for pesticide analysis has at least $3 \mathrm{~g}$ of material; sample the honey, wax, and bee bread at least 2 or 3 different points in each hive and combine those subsamples for each hive.

2. Shortly after the evaluation, such as the next day, remove any broodless frames containing honey and nectar and replace those frames with either foundation or empty drawn comb to facilitate syrup storage.

NOTE: The objective is to increase both empty storage capacity and demand for nectar. If treatment is to be applied mixed in a protein supplement, removal of food frames may not be necessary.

3. Ensure the hive has a feeder. As soon as possible after the pre-treatment evaluation, apply the treatment by pouring the syrup (step 4.4) into the feeder as rapidly as possible. Take care not to spill any syrup outside the hive where bees from other colonies might find it. NOTE: Internal feeders may work best for reducing robbing; some pesticides affect adult bee activity and treated hives may be less vigilant in protecting feeders from robbing bees ${ }^{9}$.

\section{Analysis of Hive Frame Photographs}

NOTE: Frame photographs are made during each hive evaluation. Information can be extracted from the photographs using the following protocol.

1. Activate an image analysis software, such as ImageJ, that allows for selecting a shape on a photograph with a tracing tool and measuring the area of the selected shape.

2. Retrieve a digital photograph of a frame. Select 'File' and click 'Open'. Navigate to the folder where the photographs are kept to select a photo for analysis. The image will now be opened within the ImageJ program, and the ImageJ toolbar will be active.

3. Select the 'Polygon' tool from the toolbar. Use the mouse to click on each corner to define the area of the frame covered with comb. The shape will snap together when the starting corner is reached.

4. Once the comb area is defined using the polygon tool, click on 'Analyze' and select 'Measure'; a new dialog box will pop up with the measurement. Use the column 'Area' to measure the pixels within the defined area that was drawn.

NOTE: The first time the program is used, define the desired results to be reported.

1. Click on 'Results' in the Results dialog box, and select 'Set Measurements'. Check area, and uncheck all others.

5. To define the area covered by capped brood, select the 'Freehand' tool. Depress the button on the mouse at a starting point and trace around the brood. When the starting point is reached, release the mouse button; the shape will snap into place.

1. Click 'Analyze' and select 'Measure'. If the brood pattern is not solid, due to factors such as disease, use the 'Multi-point' selection tool to count the cells instead. Convert cell counts directly to $\mathrm{cm}^{2}$ for ease of comparison; there are typically about $4.01 \mathrm{cells} / \mathrm{cm}^{2} 20$.

6. Divide the value of the traced brood shape by the value of the interior of the frame to obtain a percent frame coverage of capped brood.

7. Measure the interior of representative frames to obtain a standard so the brood surface area can be converted to $\mathrm{cm}^{2}$. NOTE: A typical Langstroth deep frame has a surface area of about $880 \mathrm{~cm}^{220}$. For example, if $20 \%$ of the interior of a Langstroth deep frame is found to be covered by brood, then the surface area would be $0.20 \times 880 \mathrm{~cm}^{2}=176 \mathrm{~cm}^{2}$.

8. Sum the brood surface area for the hive. To convert this measure to mass, multiply the surface area by $0.77 \mathrm{~g} / \mathrm{cm}^{2} 20$.

9. Calculate the surface area of honey, uncapped nectar, and stored pollen, for example, using the method described in steps 6.3 to 6.6 . NOTE: If nectar and pollen are considered together as "food stores" this reduces the components of a frame to two (food stores and brood). A typical Langstroth frame with plastic foundation and empty drawn comb weighs about $556 \mathrm{~g}^{20}$, so to calculate the weight of food stores, sum the weight of the frame with empty drawn comb and the weight of the brood mass, and subtract that weight from the observed weight of the frame in question.

\section{Representative Results}

Honey bee colony growth and phenology, measured using regular hive inspections with continuous weight monitoring, revealed significantly lower brood production among colonies exposed to imidacloprid at $100 \mathrm{ppb}$.

Hive inspections showed that adult bee masses were not significantly affected by exposure to both sublethal concentrations of 5 and 100 $\mathrm{ppb}$, but analysis of photographs of brood frames revealed that brood production in the $100 \mathrm{ppb}$ treatment was significantly lower (Figure 1). Continuous hive weight data showed different colony growth rates among groups of hives exposed to different concentrations of imidacloprid. The continuous hive weight data were divided into two parts: the $25 \mathrm{~h}$ running average data, which is related to colony growth and foraging success, and difference between the hourly raw data and the $25 \mathrm{~h}$ running average, the hourly detrended data. The daily amplitudes of the detrended data are related to foraging activity ${ }^{19,20}$ (Figure 2). While weighing hives occasionally may have detected average weight differences at some point, continuous weighing provided detrended data for information on colony behavior.

The top rail of the middle frame in a typical commercial hive is an effective location for temperature sensors.

Adult worker bees in colonies generate and maintain high temperatures $\left(33-36^{\circ} \mathrm{C}\right)$ especially in the presence of brood ${ }^{14}$ and the cluster itself is mobile to take advantage of food stores in different parts of the hive ${ }^{25,26}$. To reduce heat loss, clusters avoid having a large contact surface with exterior parts of the hive, such as the sides or bottom, so the distance from the top center of the box to the cluster is seldom large. The top center of the brood box has been shown to have reduced temperature variability compared to other locations in the hive, such as the top center of an outer frame and the top of a second box ("super") in summer and fall conditions ${ }^{20}$. In winter conditions, in which the difference between the ambient temperature and the cluster would be expected to be the greatest, the same location was found to have the highest temperature and lowest variability compared to ambient conditions, indicating the greatest contribution from the cluster itself (Figures 3 and $\mathbf{4}$; Table 1). Differences in temperature regimes could not be attributed to differences in the adult bee mass (Table 2). 
Amplitudes of sine curves fit to continuous temperature data were significantly higher in the $100 \mathrm{ppb}$ treatment than in the $\mathbf{5 p p b}$ treatment while neither treatment group was significantly different from the control group ${ }^{9}$.

Amplitudes are directly proportional to temperature variability, so the higher amplitudes indicate more variable temperatures at that point in the hive. Lower amplitudes reflect the higher brood production in the $5 \mathrm{ppb}$ and control treatment groups compared to the $100 \mathrm{ppb}$ treatment group (Figure 5). These results, combined with those from the hive inspections and continuous hive weight data, confirm that colony-level behavior was significantly affected by exposure to $100 \mathrm{ppb}$ imidacloprid.
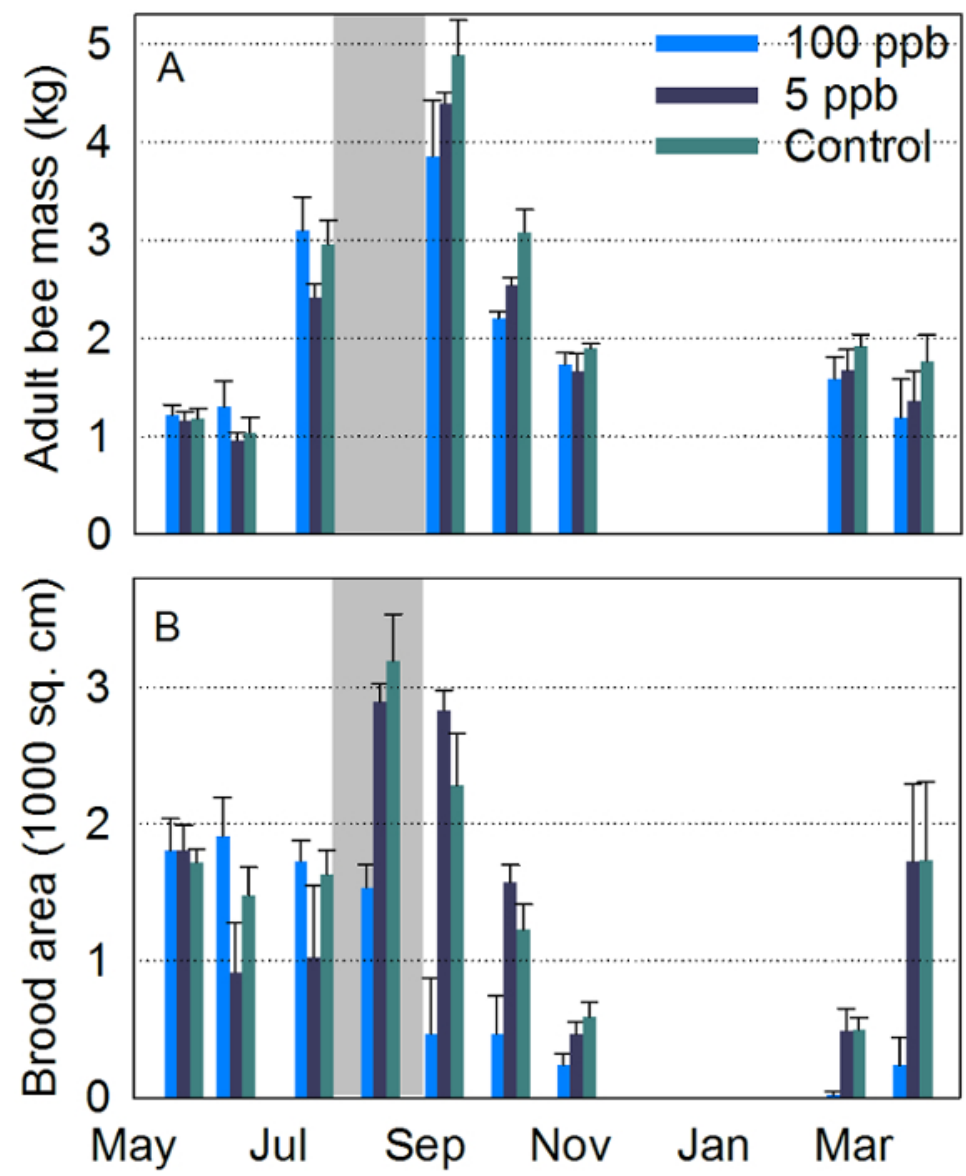

Figure 1: Adult bee and brood measures. Hive inspection data from May, 2014, to March, 2015, for colonies exposed to syrup containing 0 (control), 5 and 100 ppb imidacloprid near Tucson, AZ. (A) Average (+ SEM) total adult bee mass; (B) Average (+ SEM) sealed brood surface area. Gray zone indicates treatment period (4-6 kg of treated sucrose syrup per week per hive for 6 weeks). Four colonies per treatment group; one colony in the $100 \mathrm{ppb}$ died during winter. Adult bee masses were not recorded in August. Data here was previously published and is used here with permission ${ }^{9}$. Please click here to view a larger version of this figure. 


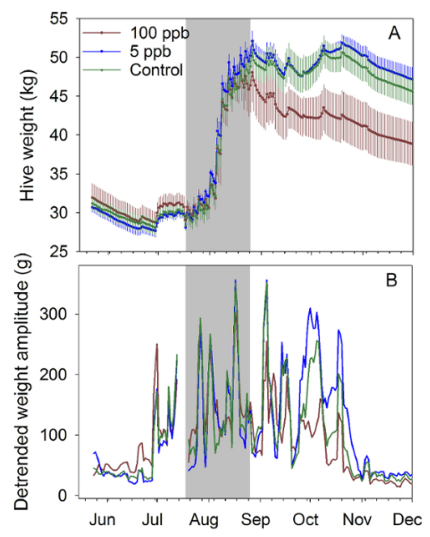

Figure 2: Honey bee hive weight data. Continuous weight data from June to December, 2014, for honey bee colonies exposed to syrup containing $\mathbf{0 , 5}$ and 100 ppb imidacloprid near Tucson, AZ. (A) Total hive weight ( \pm SEM); (B) Amplitudes of sine curves fit to detrended weight data from the same period. Gray zone indicates treatment period (4-6 kg of treated sucrose syrup per week per hive for 6 weeks). Four colonies per treatment group; one colony in the $100 \mathrm{ppb}$ died during winter. Data here was previously published and is used here with permission $^{9}$. Please click here to view a larger version of this figure.

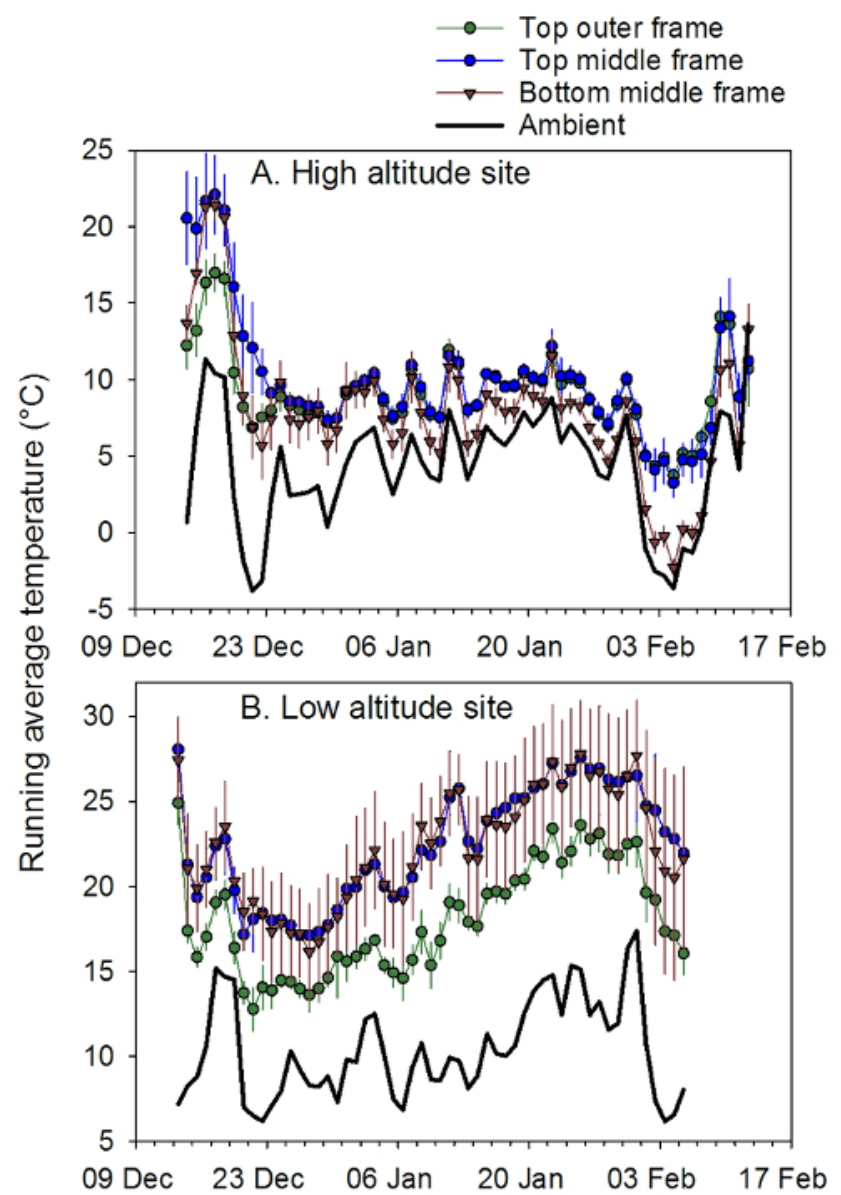

Figure 3: Within-hive temperatures at different locations. Daily average $( \pm \mathrm{SEM})$ temperatures $\left({ }^{\circ} \mathrm{C}\right)$ for 3 sensor locations inside honey bee hives. (A) 4 single-box hives kept at a high altitude site (MLEM: $2412 \mathrm{~m}$ ); and (B) 3 hives kept at a lower altitude site (SRER: $719 \mathrm{~m}$ ) near Tucson, AZ from December 2013 to February 2014. Hives were wooden Langstroth deep boxes (43.65 I capacity) fitted with wooden inner covers and metal-lined telescoping lids. Thermocouple temperature probes were attached to the top bars of the frames, and an integrated datalogger/temperature sensor was placed on the bottom rail of the middle frame. These data show that the sensor position at the top of the middle frame was consistently higher than ambient compared to the other sensor positions and thus informative about temperature management by the bee colonies. Please click here to view a larger version of this figure. 


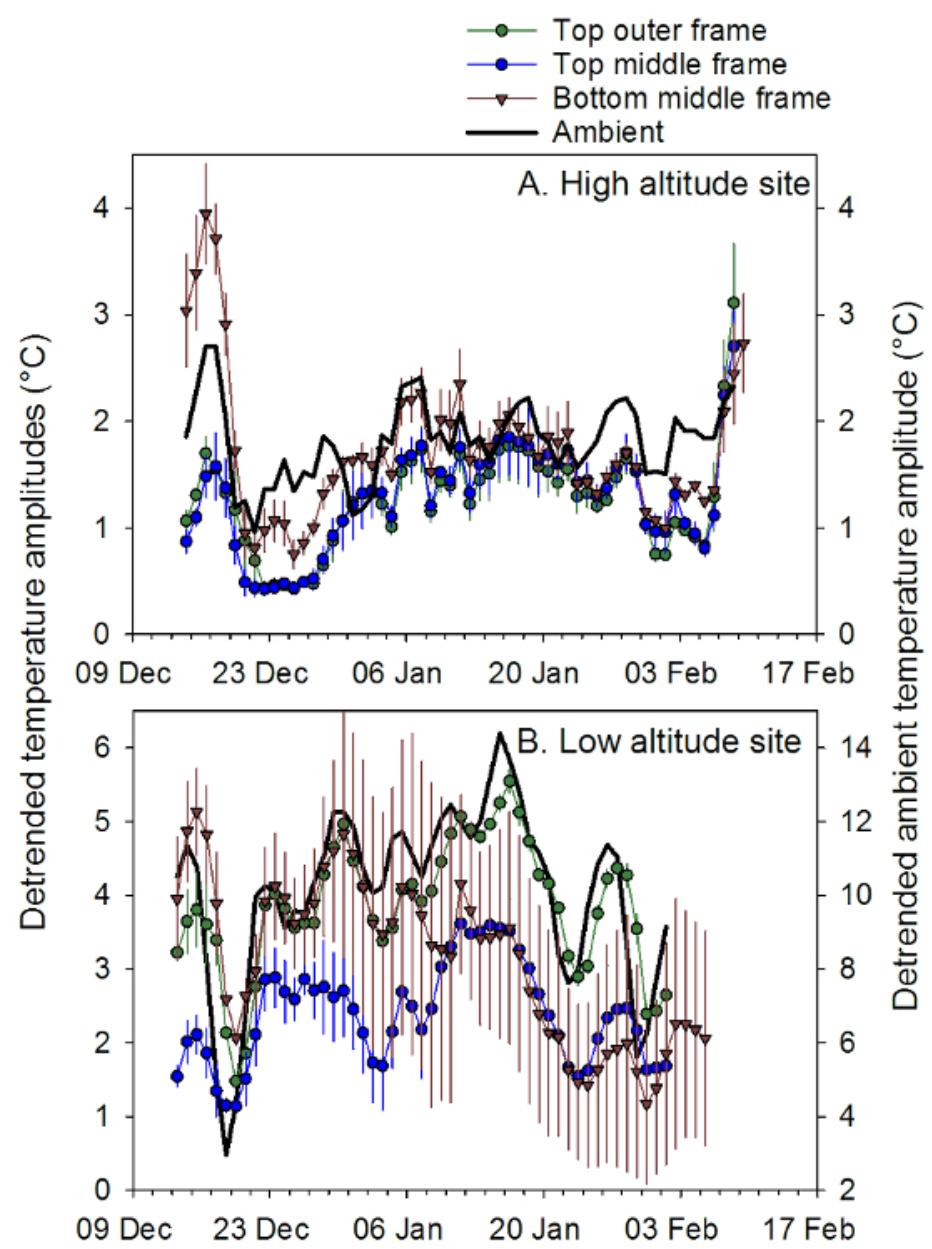

Figure 4: Within-hive temperature variability at different locations. Daily average ( \pm SEM) amplitudes for sine curves fit to hourly detrended temperature data for 3 sensor locations inside honey bee hives. (A) 4 hives kept at at high altitude site (MLEM: $2412 \mathrm{~m}$ ); and (B) 3 hives kept at a lower altitude site (SRER: 719 m) near Tucson, AZ from December 2013 to February 2014. Hives were wooden Langstroth deep boxes (43.65 I capacity) fitted with wooden inner covers and metal-lined telescoping lids. Thermocouple temperature probes were attached to the top bars of the frames, and an integrated datalogger/temperature sensor was placed on the bottom rail of the middle frame. These data show that the sensor position at the top of the middle frame was consistently lower in variability (here measured as the amplitude of sine curves fit to hourly detrended data) than ambient compared to the other sensor positions and thus informative about temperature management by the bee colonies. Scales for the ambient temperature (solid black line) are on the right of each graph. Please click here to view a larger version of this figure. 

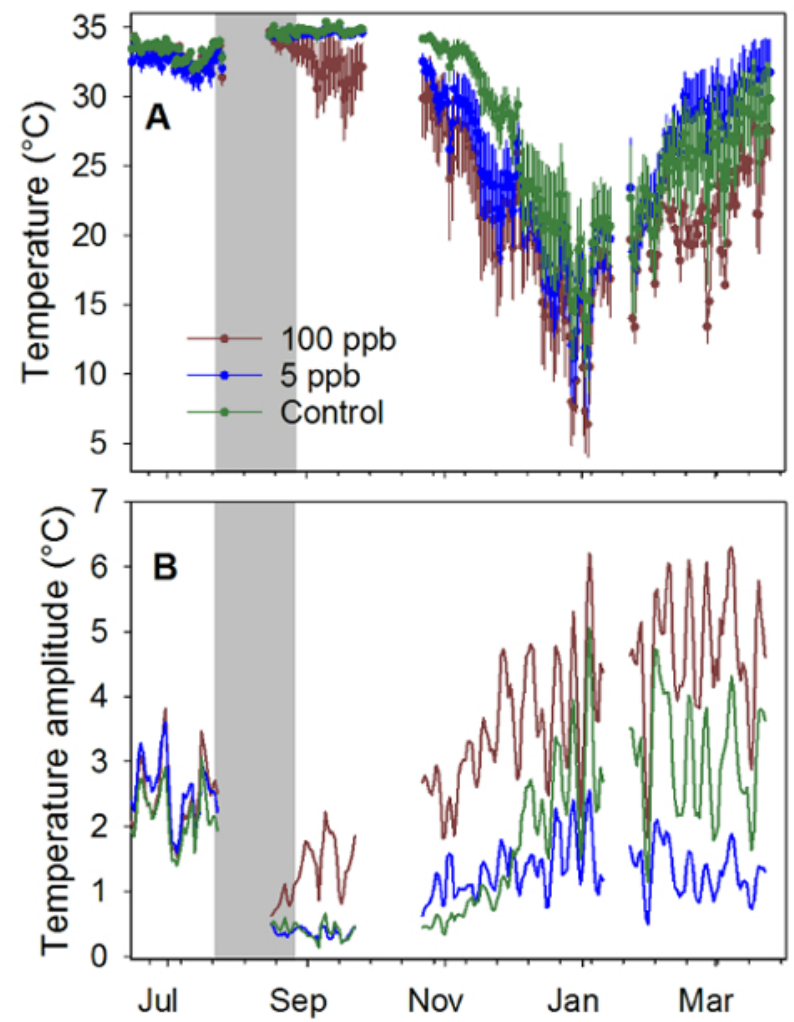

Figure 5: Within-hive temperatures for treated colonies. Continuous temperature data from June to December, 2014, for honey bee colonies exposed to syrup containing 0,5 and $100 \mathrm{ppb}$ imidacloprid near Tucson, AZ. (A) Average hive temperature ( \pm SEM); (B) Amplitudes of sine curves fit to detrended temperature data from the same period. Gray zone indicates treatment period (4-6 kg of treated sucrose syrup per week per hive for 6 weeks). Four colonies per treatment group; one colony in the $100 \mathrm{ppb}$ died during winter. Data here was previously published and is used here with permission (Meikle et al. 2016a). Please click here to view a larger version of this figure.

\begin{tabular}{|l|l|l|l|l|}
\hline Comparison & t test & adj. P & Site & Avg. diff. $\left({ }^{\circ} \mathrm{C}\right)$ \\
\hline Pos. 2 - Pos. 1 & 2.05 & 0.0485 & MLEM & $1.00 \pm 0.25$ \\
\hline & & & SRER & $4.38 \pm 0.12$ \\
\hline \hline Pos. 2 - Pos. 3 & 0.84 & 1 & MLEM & $2.05 \pm 0.21$ \\
\hline & & & SRER & $0.12 \pm 0.11$ \\
\hline
\end{tabular}

Table 1: Average temperature differences and post hoc comparisons among different sensor positions within honey bee hives. Position 1: top outer frame; Position 2: top middle frame; Position 3: bottom middle frame. 4 single-box hives were kept at a high altitude site (MLEM: $2412 \mathrm{~m}$ ) and 3 hives were kept at a lower altitude site (SRER: $719 \mathrm{~m}$ ) near Tucson, AZ from December 2013 to February 2014 . Hives were wooden Langstroth deep boxes (43.65 I capacity) fitted with wooden inner covers and metal-lined telescoping lids. Thermocouple temperature probes were attached to positions 1 and 2, and an integrated datalogger/temperature sensor was attached to position 3 .

\begin{tabular}{|c|c|c|c|}
\hline Group & Date & $\begin{array}{l}\text { Average }( \pm \text { SEM) adult bee mass } \\
\text { (g) }\end{array}$ & $\begin{array}{l}\text { Average }( \pm \text { SEM) brood surface } \\
\text { area }\left(\mathrm{cm}^{2}\right)\end{array}$ \\
\hline MLEM & 18 Nov. 2013 & $2119 \pm 412$ & $1372 \pm 396$ \\
\hline SRER & 15 Nov. 2013 & $2270 \pm 312$ & $53 \pm 30$ \\
\hline MLEM & 13 Feb. 2014 & $2171 \pm 105$ & 0 \\
\hline SRER & 11 Feb. 2014 & $2027 \pm 487$ & $867 \pm 79$ \\
\hline
\end{tabular}

Table 2: Average adult bee masses and brood masses, estimated using the protocols described here, for four hives kept at a high altitude site (MLEM: $2412 \mathrm{~m}$ ); and three hives kept at a lower altitude site (SRER: $719 \mathrm{~m}$ ) near Tucson, AZ from December 2013 to February 2014. Hives were wooden Langstroth deep boxes (43.65 I capacity) fitted with wooden inner covers and metal-lined telescoping lids. Thermocouple temperature probes were attached to positions 1 and 2 , and an integrated datalogger/temperature sensor was attached to position 3. 


\section{Discussion}

Just as a crop scientist requires accurate and precise data to evaluate the effects of different treatments on plant growth and yield, honey bee researchers require accurate and precise data to evaluate bee colony growth and activity. These kinds of data are particularly important when the treatment effects may be subtle and long term, such as might be expected when bees are exposed to low concentrations of pesticides.

Continuous hive weight data contain much information on the growth and activity of the colonies being studied, as well as the response of those colonies to exogenous disturbances, such as from robbing bees and from the addition of sugar syrup to a feeder ${ }^{9}$. However, proper interpretation of continuous weight and temperature data requires some knowledge of the size of the colony on a periodical basis. While the size of the adult bee population can be estimated from the daily amplitudes of detrended hourly data during a nectar flow, due to the increased forager traffic, outside of a nectar flow the relationship does not hold well ${ }^{20}$. Likewise, colony weight changes due to foraging success, food consumption, and bee population increases and decreases (e.g. bee kills) are partly a function of colony size. Smaller colonies may be healthy but show lower growth rates and less forager mass, for example, simply due a smaller forager population.

Identifying a consistent and informative fixed position for a temperature sensor within the hive is crucial for producing reproducible results, particularly with overwintering. Previous research has shown that temperature monitoring at the top rail of a central frame in the brood (bottom) box were less correlated with ambient (external) temperatures than several other positions in the hive and were more influenced by the colony itself $^{20}$. During winter, temperature differences between the colony bee cluster and ambient would expected to be high, and so differences among positions within the hive would likewise be expected to be greater than during warmer months. In addition, the bee cluster will move to different locations within the hive to exploit food resources ${ }^{25,26}$. The winter study described here showed that temperatures at horizontal positions, from the top middle to top outer frames, were significantly different while vertical differences on the middle frame were not. Horizontal air movement across frames is relatively restricted while vertical movement along a frame is not, so vertical mixing would be expected to be greater. These results confirm that a sensor position on the top rail of the middle frame is likely to be informative with respect to temperature influences from the colony.

Hive evaluations as they are described here resolve some of these issues. By subtracting the sum of the hive parts from the total hive weight measured during inactivity, such as at night, the estimate of the adult bee mass is independent of the number of foraging bees and thus insensitive to the time of evaluation. Recording data from a scale allows little room for subjectivity, and frame photographs provide a permanent record, so analyses of brood or food surface areas can be revisited if necessary, thus reducing the dependence on the training of the person doing the analyses.

The methods described here are not without sources of error. Hive parts need to be as free of bees as possible, and any significant pieces of comb or other material that is removed should be weighed before discarded. The moisture content of wooden components can change over time and empty hives can exhibit measurable daily oscillations in weight due to daily changes in ambient relative humidity ${ }^{24}$. A hive containing a colony will probably not change as much because honey bees tend to maintain a relatively constant humidity inside the hive ${ }^{27}$, which would attenuate the ambient effects. These methods also depend on working rapidly. Once a hive is taken apart, the temperature and gas conditions change drastically and cool temperatures can impact brood health ${ }^{14}$. Taking apart a hive without a screen to keep out bees from neighboring colonies can promote robbing, particularly in times of nectar dearth.

Modifications of these procedures are occasionally necessary. While it is in the interest of the researchers to gather as much data as possible, on some occasions, such as when many hives must be inspected, when robbing pressure is high, or when weather conditions are not optimal, workers must move quickly and forgo some data. If data on food reserves are not crucial, frames containing only honey and/or pollen, or frames with only wax or foundation, should be weighed but not photographed. Levels of uncapped larvae and eggs are also of interest to some researchers and they are often detected in frame photographs; however, in order to avoid sampling bias a researcher must be confident in being able to detect them in all photographs. Often only capped brood can be reliably and consistently identified on photographs taken under field conditions. Regarding the application of treatment, some hives, particularly those fed higher doses of imidacloprid, can become lethargic, causing a reduction in both their consumption of syrup and their ability to defend their hive. If a colony fails to consume its syrup after 5-7 $d$, the syrup should be removed, weighed, and discarded (to avoid contamination due to fermentation) and the colony offered fresh syrup. By weighing the discarded syrup, researchers will have record of how much each colony consumed, and thus can calculate total dosage. Finally, the application of treatments in sugar syrup is most effective when there are few or no alternative nectar sources. Alternative nectar sources would dilute the treatment to an unknown degree. Researchers should bear in mind, as was pointed out in the protocol section, that pesticides such as imidacloprid are associated with reduced activity levels, including hive protection, depending on the concentration. Reduced colony defense may also lead to increased robbing and possible confounding of treatments.

The results presented here show that continuous monitoring of hive weight and internal temperature are sensitive to colony-level behaviors, even with respect to behavioral changes induced by very low $(5 \mathrm{ppb})$ concentrations of a neurotoxic pesticide. Further application of these methods to other neurotoxins and to pesticides with other modes of action, such as insect growth regulators, would increase our understanding of the effects of field-realistic doses on honey bee colonies.

\section{Disclosures}

The authors have no competing financial interests. 


\section{Acknowledgements}

The authors warmly thank K. Anderson, J.J. Adamczyk, E. Beren, I Carstensen, M. Giansiracusa B. Mott, N. Holst and A.R. Stilwell for discussions on design and execution and for help in the field.

\section{References}

1. Krupke, C.H., Hunt, G.J., Eitzer, B.D., Andino, G., Given, K. Multiple routes of pesticide exposure for honey bees living near agricultural fields PLOS ONE. 7(1), e29268 (2012).

2. Pochi, D., Biocca, M., Fanigliulo, R., Pulcini, P., Conte, E. Potential exposure of bees, Apis mellifera L., to particulate matter and pesticides derived from seed dressing during maize sowing. Bull. Environ. Contam. Toxicol. 89, 354-361 (2012).

3. Reetz, J., Zühlke, S., Spiteller, M., Wallner, K. Neonicotinoid insecticides translocated in guttated droplets of seed-treated maize and wheat: a threat to honeybees? Apidologie. 42, 596-606 (2011).

4. Stoner, K.A., Eitzer, B.D. Movement of soil-applied imidacloprid and thiamethoxam into nectar and pollen of squash (Cucurbita pepo). PLoS ONE. 7(6), e39114 (2012).

5. Zhu, Y.C., et al. Spray toxicity and risk potential of 42 commonly used formulations of row crop pesticides to adult honey bees (Hymenoptera: Apidae). J. Econ. Entomol. 1-8 (2015).

6. Mullin, C.A., et al. High levels of miticides and agrochemicals in North American apiaries: implications for honey bee health. PLoS ONE. 5, e9754 PMID: 20333298 (2010).

7. Cresswell, J.E. A meta-analysis of experiments testing the effects of a neonicotinoid insecticide (imidacloprid) on honey bees. Ecotoxicol. 20, 149-157 (2011).

8. Dively, G.P., Embrey, M.S., Kamel, A., Hawthorne, D.J., Pettis, J.S. Assessment of chronic sublethal effects of imidacloprid on honey bee colony health. PLoS ONE. 10(3), e0118748 (2015).

9. Meikle, W.G., et al. Sublethal effects of imidacloprid on honey bee colony growth and activity at three sites in the U.S. PLoS ONE. 11(12), e0168603 (2016).

10. Johnson, B.R. Within-nest temporal polyethism in the honey bee. Behav. Ecol. Sociobiol. 62(5), 777-784 (2008).

11. Moritz, R.F.A., Southwick, E.E. Bees as superorganisms: An evolutionary reality., Springer, Heidelberg, Germany (1992).

12. Schmolz, E., Lamprecht, I., Schricker, B. A method for continuous direct calorimetric measurements of energy metabolism in intact hornet (Vespa crabro) and honeybee (Apis mellifera) colonies. Thermochem. Acta. 251, 293-301 (1995).

13. Southwick, E.E., Mugaas, J.N. A hypothetical homeotherm: The honey bee hive, Comp. Biochem. Physiol. 40A, 935- 944 (1971).

14. Stabentheiner, A., Kovac, H., Brodschneider, R. Honeybee colony thermoregulation - regulatory mechanisms and contribution of individuals in dependence on age, location and thermal stress. PLoS ONE. 5(1), e8967 (2010).

15. Delaplane, K.S., van der Steen, J., Guzman-Novoa, E. Standard methods for estimating strength parameters of Apis mellifera colonies. In Dietemann V.,Ellis JD, Neumann P (Eds) The COLOSS BEEBOOK, Volume I: standard methods for Apis mellifera research. J. Apic. Res. 52(1) (2013).

16. Beekman, M., Ratnieks, F.L.W. Long-range foraging by the honey-bee, Apis mellifera L. Functional Ecology., 14, 490-496 (2000).

17. Buchmann, S.L., Thoenes, S.C. The electronic scale honey bee colony as a management and research tool, Bee Sci. 1, 40-47 (1990).

18. Lecocq, A., Kryger, P., Vejsnæs, F., Bruun Jensen, A. Weight watching and the effect of landscape on honeybee colony productivity: Investigating the value of colony weight monitoring for the beekeeping industry. PLoS ONE. 10(7), e0132473 (2015).

19. Meikle, W.G., Rector, B.G., Mercadier, G., Holst, N. Within-day variation in continuous hive weight data as a measure of honey bee colony activity. Apidologie. 39, 694-707 (2008).

20. Meikle, W.G., Weiss, M., Stilwell, A.R. Monitoring colony phenology using within-day variability in continuous weight and temperature of honey bee hives. Apidologie. 47, 1-14 (2016).

21. Meikle, W.G., Holst, N. Application of continuous monitoring of honey bee colonies. Apidologie. 46, 10-22 (2015).

22. De Smet, L., et al. Stress indicator gene expression profiles, colony dynamics and tissue development of honey bees exposed to sub-lethal doses of imidacloprid in laboratory and field experiments. PLoS ONE. 12(2), e0171529 (2017).

23. Danka, R.G., Rinderer, T.E., Hellmich, R.A. II, Collins, A.M. Foraging population sizes of African and European honey bee (Apis mellifera L.) colonies. Apidologie. 17(3), 193-202 (1986).

24. Meikle, W.G., Holst, N., Mercadier, G., Derouané, F., James, R.R. Using balances linked to dataloggers to monitor honeybee colonies, J. Apic. Res. 45(1), 39-41 (2006).

25. Owens, C.D. The thermology of wintering honey bee colonies. United States Department of Agriculture, Agricultural Research Service, Technical Bulletin no. 1429 (1971).

26. Szabo, T.I. Thermology of wintering honey-bee colonies in 4-colony packs. Am. Bee J. 189, 554-555 (1989).

27. Human, H., Nicolson, S.W., Dietemann, V. Do honeybees, Apis mellifera scutellata, regulate humidity in their nest? Naturwissenschaften. 93, 397-401 (2006). 